\title{
Cierre secuencial de la pared abdominal Una nueva técnica quirúrgica diseñada en el Centenario Hospital Miguel Hidalgo para el manejo del abdomen abierto
}

Flores-Álvarez E*, Rivera-Barragán V**, De la Torre-González JC**

\section{Resumen}

- El abdomen abierto es una técnica quirúrgica emplea-

- da en el tratamiento de los pacientes con procesos

- infecciosos peritoneales severos, en el trauma abdomi-

- nal grave en pacientes que requieren cirugía de control

- de daños y en el manejo del síndrome de hipertensión

- abdominal.

- El tratamiento con abdomen abierto ocasiona riesgos

- trascendentales para el paciente tales como alteracio-

- nes hidroelectrolíticas, contaminación externa de ór-

- ganos abdominales, desarrollo de fístulas intestinales,

- adherencias obstructivas, defectos herniarios gigantes

- de la pared abdominal y la presencia de grandes

- heridas cruentas con cicatrización lenta, con conse-

- cuencias estéticas y funcionales importantes. Existen

- técnicas que además de manejar el abdomen abierto

- tratan de evitar la retracción aponeurótica y el desa-

- rrollo de hernias ventrales gigantes que requieren de una compleja reconstrucción tardía de la pared abdominal. El Cierre Secuencial de la Pared Abdominal es una técnica desarrollada en el Centenario Hospital Miguel Hidalgo en la que se emplean materiales y métodos utilizados en diversas técnicas del manejo de abdomen abierto, adecuados a la infraestructura del hospital y a la disponibilidad de materiales. Los beneficios del procedimiento propuesto son reparar, drenar, descomprimir y ofrecer control de la pared abdominal en aquellos pacientes que requieren tratamiento con abdomen abierto. La técnica tiene la ventaja de que el paciente puede permanecer en su cama, sin necesidad de reiterados ingresos a quirófano y debido a que se trata de una técnica sencilla y fácil de realizar así como por el uso de materiales inertes se asocia a un bajo índice de complicaciones y resultados iniciales alentadores. LUXMÉDICA 5(15): 15-22

Palabras clave: Abdomen abierto, pared abdominal, hipertensión abdominal, hernias ventrales.

* Cirujano general, Cirujano oncólogo, Maestro en ciencias.

** Cirujano general, Departamento de Enseñanza e Investigación y Servicio de Cirujía General. Centenario Hospital Miguel Hidalgo. Ags, Ags.

Fecha de recibido: 14-junio-2010

Fecha de aceptado: 24-agosto-2010

Correspondencia: Flores Alvarez E. Maestro en Ciencias Médicas. Departamento de Cirugía. Centenario Hospital Miguel Hidalgo.

Galeana Sur \#465 Col. Obraje. Tel 994-67-20. Correo electrónico: efflorez@hotmail.com 


\section{Introducción}

El abdomen abierto es una técnica quirúrgica utilizada en el tratamiento de los pacientes con sepsis severa de origen abdominal y empleada recientemente en pacientes con traumatismo abdominal grave que requieren laparotomía abreviada o de salvamento y en la prevención y tratamiento del síndrome de hipertensión abdominal ${ }^{1}$.

El tratamiento con abdomen abierto implica muchas desventajas para el paciente, tales como alteraciones hidroelectrolíticas, alto riesgo de contaminación de órganos intraabdominales, desarrollo de fístulas intestinales, adherencias obstructivas, defectos herniarios gigantes de la pared abdominal y la presencia de grandes heridas cruentas con cicatrización lenta, con consecuencias estéticas y funcionales importantes ${ }^{2,3}$.

En la actualidad hay un notable aumento en el empleo de las técnicas de abdomen abierto, así mismo se han ideado nuevos procedimientos que además de resolver el evento agudo en forma satisfactoria, intentan evitar las serias complicaciones inherentes al manejo con un abdomen abierto. El Cierre Secuencial de la Pared Abdominal (CSPA) es una técnica desarrollada en el Centenario Hospital Miguel Hidalgo en la que se emplean materiales y métodos utilizados en diversas técnicas del manejo de abdomen abierto, adecuados a la infraestructura del hospital y a la disponibilidad de materiales.

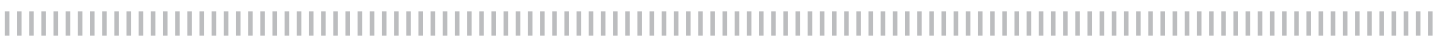

\section{Antecedentes históricos}

Price $^{4}$ y Torek $^{5}$, a principios del siglo pasado demostraron la efectividad del desbridamiento y lavado de la cavidad peritoneal en pacientes con apendicitis aguda, reduciendo en forma significativa la mortalidad posoperatoria. Artz ${ }^{6}$, en 1960 utilizó la irrigación durante el acto quirúrgico con antibióticos y Schumer ${ }^{6}$ la irrigación continua posoperatoria intraperitoneal. Huspeth $^{7}$ propuso el desbridamiento radical de la superficie peritoneal tanto visceral como parietal.

Steimberg, en los años 70 propuso el tratamiento de la cavidad peritoneal considerándola como un gran absceso, dejándola abierta por 48-72 horas, con lo cual redujo a tan sólo un $7 \%$ la mortalidad de los pacientes con peritonitis supurativa.

Bajo la premisa de que no es suficiente dejar el abdomen abierto sino que deben drenarse las colecciones adecuadamente, Teichmann y Wittmann ${ }^{9}$ en Alemania en 1980, sugirieron la utilización del régimen de exploración manual diaria y el lavado de la cavidad abdominal abordándolo a través de una cremallera insertada en una malla de polipropileno.

En 1984, en el Hospital San Juan de Dios de Bogotá Colombia, el Dr. Oswaldo Borraéz ${ }^{10}$ utilizó por primera vez una bolsa de polivinilo para cubrir el abdomen abierto de un paciente con infección abdominal. Esta técnica conocida como "Bolsa de Bogotá", representa el método quirúrgico más utilizado para el manejo del abdomen abierto hasta nuestros días.

El tratamiento del abdomen abierto en trauma, remonta sus orígenes al manejo de los heridos durante la segunda guerra mundial. Ogilvie ${ }^{11}$, utilizó mantas de algodón esterilizadas y lubricadas con vaselina, sutu- 
radas con catgut a los bordes de la aponeurosis. En su escrito, enfatiza la trascendencia de evitar el crecimiento del defecto, la protección de los órganos abdominales y la importancia de una pared abdominal íntegra para preservar la mecánica ventilatoria.

\section{Abdomen Abierto}

El empleo de la técnica quirúrgica del abdomen abierto va en aumento al tiempo que los cirujanos se familiarizan cada vez más con el procedimiento, su mecanismo de acción, sus ventajas y desventajas. Hasta hace dos décadas, la idea de que un paciente abandonara la sala de operaciones con el abdomen abierto de manera planeada era algo inadmisible. Esta idea ha cambiado radicalmente, gracias a un mejor entendimiento y experiencia en la cirugía de control de daños y la reintervención, aunado a un mayor conocimiento de las repercusiones del síndrome compartimental abdominal. Sabemos que el edema visceral y retroperitoneal debido al estado de choque y reperfusión tisular, puede incrementar la presión intraabdominal a niveles peligrosos, ocasionando una disfunción orgánica sistémica. Se ha demostrado que pacientes en estas condiciones tienen una mejor evolución si son tratados con abdomen abierto temporalmente, permitiendo una mejor perfusión visceral abdominal y funcionamiento sistémico. ${ }^{12,13}$ Se reconoce que la combinación de acidosis, coagulopatía e hipotermia representa un estado que debe ser evitado o corregido con la terminación rápida de una operación encaminada esencialmente a controlar un sangrado que pone en riesgo la vida o a limitar la contaminación peritoneal por lesiones viscerales. Este escenario permite una operación definitiva en mejores condiciones una vez lograda la reversión de la tríada letal y la estabilización del paciente en una unidad de terapia intensiva ${ }^{14}$.

Los principios de la terapia quirúrgica para la infección intraabdominal han sido revisados por el Dr. Wittmann ${ }^{15}$, quien define cuatro principios fundamentales que el cirujano debe considerar para lograr reducir la morbi-mortalidad asociada a la infección intraabdominal:

1 Eliminar o controlar la fuente de la infección. Si este principio no es logrado mediante el acto quirúrgico, todas las medidas de manejo médico serán inútiles.

2 Limpiar en forma exhaustiva la cavidad abdominal infectada, aspirando todos los líquidos infectados.

3 Prevenir o tratar el síndrome del compartimento abdominal mediante el manejo del abdomen abierto facilitando la frecuente reexploración. Esta maniobra es fundamental para liberar la hipertensión abdominal causada por el edema peritoneal y el secuestro de líquidos, de tal manera que se evitan las consecuencias nocivas del síndrome compartimental abdominal.

4 Prevenir y tratar la infección persistente o recurrente mediante la relaparotomía planeada, decisión que debe tomarse antes o durante la primera intervención para tratar la peritonitis. Es necesario reexplorar la cavidad abdominal, evacuar, desbridar o resecar cuantas veces sea necesario hasta obtener una cavidad abdominal libre de infección.

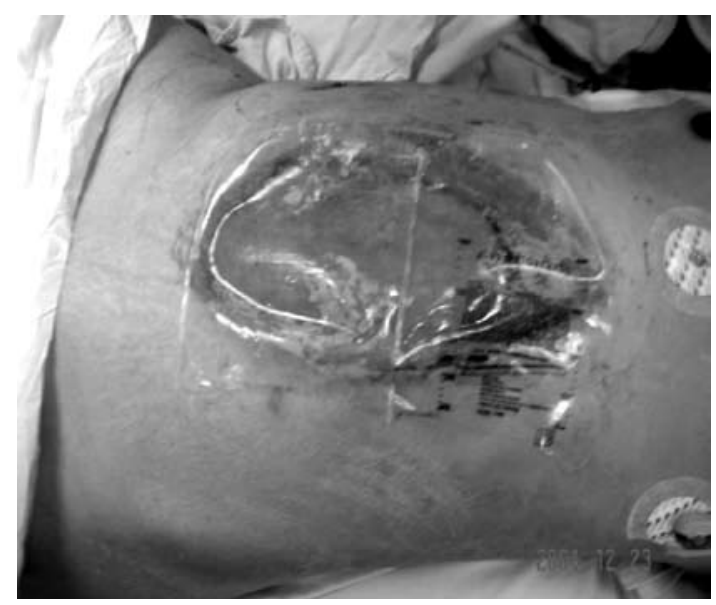

Figura 1. Paciente con abdomen abierto. 


\section{Indicaciones para Abdomen Abierto}

Las indicaciones para la utilización de la estrategia quirúrgica del abdomen abierto se pueden dividir en 4 grupos:

I Abdominoviscerales. Peritonitis aguda supurativa generalizada, abscesos peritoneales múltiples, absceso pancreático, pancreatitis aguda con necrosis infectada grave, dehiscencia de suturas intestinales, fístulas intestinales y duda de la viabilidad intestinal.

II Parietales. Evisceración con retracción de los bordes de la pared abdominal, infección de la pared abdominal y necrosis de la pared abdominal.

III Generales. Choque séptico y compromiso metabólico o nutricional intenso.

IV Trauma abdominal grave. Cirugía de control de daños y síndrome compartimental abdominal.

Varios investigadores han demostrado que un cierre forzado y bajo tensión de la cavidad abdominal puede ocasionar isquemia, necrosis, tendencia a la infección y dehiscencia de la pared abdominal, con una mortalidad hasta del $90 \%{ }^{14,15}$. La causa principal de estos fenómenos son el aumento de la presión de la cavidad abdominal y el síndrome compartimental abdominal, caracterizados por un aumento de la presión de inspiración en la vía aérea, aumento de la presión venosa central, hi-

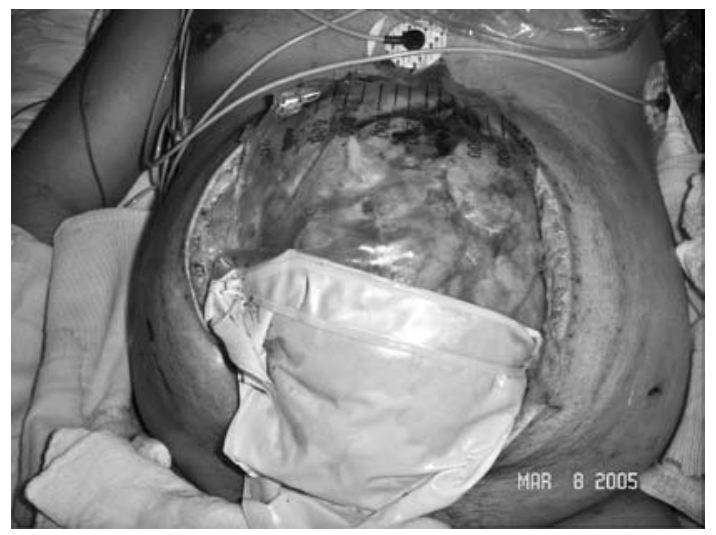

Figura 2. Paciente con abdomen abierto y bolsa de Bogotá. percapnia, hipoxia y oliguria, que en forma característica son reversibles al descomprimir la cavidad abdominal ${ }^{16}$.

\section{Técnicas de cierre progresivo de la pared abdominal}

Existen técnicas que además de manejar el abdomen abierto tratan de evitar la retracción aponeurótica y el desarrollo de hernias ventrales gigantes que requieren de una compleja reconstrucción tardía de la pared abdominal que implica a su vez una elevada morbi-mortalidad.

\section{Cierre aponeurótico inducido mediante vacío}

Consiste en colocar una bolsa de polietileno fenestrada por debajo del peritoneo cubriendo el contenido intrabdominal, sobre el plástico se colocan compresas y tubos de drenaje que se conectan a un sistema de succión y encima se coloca un plástico adhesivo de poliéster cubriendo todo el abdomen. Se asocia a desarrollo de fístulas intestinales en 17 a 30\% y una probabilidad de éxito en el cierre aponeurótico completo de $40 \%{ }^{17,18}$.

\section{Afrontamiento progresivo de bolsa de Bogotá}

Después de la descompresión abdominal se realiza un enrollamiento progresivo de la bolsa fijada a la aponeurosis, manteniendo este enrollamiento con el uso de clips, favorece la aproximación de los bordes aponeuróticos con un cierre parcial del defecto en la pared abdominal ${ }^{19}$.

\section{Retención dinámica de la pared abdominal}

Técnica descrita para el cierre gradual y completo de la pared abdominal en pacientes tratados con abdomen abierto, evitando el uso de malla para el cierre definitivo. Basa su éxito en la colocación de puntos totales de poliéster traccionables sobre una interfase de polietileno que evita el contacto con las asas intestinales y la pérdida de líquido peritoneal. Sus autores reportan un 
alto índice de cierre total de la pared y escasas complicaciones ${ }^{20}$.

\section{Cierre primario organizado de la pared abdominal}

Método que consiste en un cierre primario organizado de la pared abdominal utilizando tubos flexibles de equipos para infusión intravenosa y la interposición de una bolsa colocada libremente entre la superficie de la pared abdominal y las asas intestinales, esta técnica permite el cierre progresivo de la pared abdominal y eventualmente el cierre aponeurótico primario ${ }^{21}$.

\section{Parche de Wittmann}

Consiste en dos hojas adheribles de un material biocompatible de polímeros. Ambas hojas se fijan a la aponeurosis y se adhieren entre sí mediante velcro, cada hoja se adhiere entre sí cuando se comprimen una contra la otra y solo una fuerza mayor capaz de desgarrar la aponeurosis intacta permitiría que ambas hojas se separen. Al desprender la parte superior de la hoja de la inferior se puede ingresar a la cavidad abdominal, ambas hojas se van aproximando paulatinamente hasta que las aponeurosis pueden ser suturadas entre sí15.

Existe abundante información de los diferentes procedimientos empleados en el mundo en el tratamiento del abdomen abierto y el cierre progresivo de la pared abdominal. El grupo quirúrgico del Centenario Hospital Miguel Hidalgo se ha dado a la tarea de comprender y familiarizarse con dichos métodos para aplicarlos en nuestros pacientes. Siendo técnicas reservadas para países de primer mundo, debido a los altos costos de los insumos necesarios para su realización, su utilidad en nuestro medio resulta muy limitada.

El principal beneficio de las técnicas del cierre progresivo de la pared abdominal es el control de la pared muscular abdominal, pudiendo contar con la integridad de ésta para realizar el cierre definitivo sin el uso de materiales protésicos permanentes, que han demostrado un elevado índice de complicaciones ${ }^{22}$, particularmente el desarrollo de fístulas intestinales y su elevada morbilidad y mortalidad asociada o en el caso del uso de la Bolsa de Bogotá19, en que los pacientes son condenados a vivir con una "hernia ventral planeada" y grandes cicatrices por el cierre por segunda intención, que en el mejor de los casos se intenta reparar mucho tiempo después con procedimientos complejos y un elevado índice de fracasos ${ }^{23}$.

\section{Cierre secuencial de la pared abdominal}

Los beneficios del procedimiento propuesto son reparar, drenar, descomprimir y ofrecer control de la pared abdominal, en aquellos pacientes que requieren tratamiento con abdomen abierto. La técnica tiene la ventaja de que el paciente puede permanecer en su cama, sin necesidad de reiterados ingresos a quirófano requeridos por otros métodos. Debido a que se trata de una técnica sencilla y fácil de realizar, así como por el uso de materiales inertes se asocia a un bajo índice de complicaciones.

La principal indicación del cierre secuencial de la pared abdominal ocurre cuando la pared abdominal no puede ser afrontada sin tensión, debido al edema y distensión de asas intestinales secundarias a sepsis abdominal, sin embargo las indicaciones pueden ser múltiples:

* Cuando el abdomen no puede ser cerrado sin dejar tensión.

* Cuando la lesión intra-abdominal no puede ser resuelta adecuadamente en la intervención primaria.

* Cuando el paciente esta inestable o muy grave para realizar una operación extensa y es necesaria una cirugía de control de daños.

* Cuando la viabilidad del intestino es cuestionable y requiere una segunda intervención, conocida como cirugía de segunda mirada o second look.

* Cuando hemos realizado una anastomo- 
sis primaria en circunstancias críticas con una alta probabilidad de dehiscencia.

* Ante una hemorragia de difícil control que requiere empaquetamiento.

* Cuando la perfusión de un órgano es crítica, después de una operación extensa que ocasiona edema peritoneal por exposición.

\section{Descripción de la técnica quirúrgica}

En la técnica del cierre secuencial de la pared abdominal se emplea una interfase de polietileno para contener las vísceras abdominales, que generalmente es una bolsa recolectora de orina que se fija con 6 puntos totales de poliéster ${ }^{5}$ a la pared abdominal, tres a cada lado de la herida, e inicialmente se mantienen sin tensión y son anudados al final del procedimiento. Se debe realizar una disección amplia de los bordes de la aponeurosis separándolas del tejido celular subcutáneo creando el suficiente espacio para colocar puntos aponeuróticos a 3 o $4 \mathrm{~cm}$ del borde y entre sí, mediante la utilización de tubos de drenaje cerrado de 1/8" o catéteres intravenosos largos sin retirar la guía de acero. Enseguida se procede a realizar el afrontamiento de los bordes de la aponeurosis traccionando los tubos o catéteres y se fijan mediante un dispositivo, que pueden ser pinzas de Kelly o el sistema de cubos de acrílico con tornillos de ajuste, que fueron diseñados en el Centenario Hospital Miguel Hidalgo por los autores del estudio. Al final se ajustan y anudan los puntos totales de la bolsa de poliéster. En ocasiones se ha colocado una compresa húmeda sobre la herida y una sonda de aspiración cubiertas por una hoja de steri drape permitiendo aspirar el exceso de líquido peritoneal o secreciones intraabdominales.

A través de la medición de la presión intraabdominal ${ }^{24}$ y de la evolución clínica del paciente se determinará la frecuencia con la que se ajusta el cierre aponeurótico

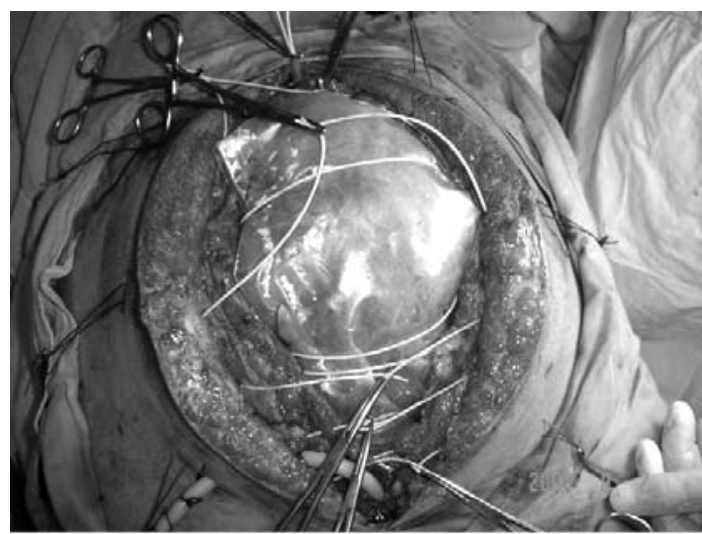

Figura 3. Cierre secuencial de la pared abdominal. Se muestra la interfase de polietileno y la fijación de los catéteres de retracción insertados en la aponeurosis.

procurando que los eventos de cierre secuencial sean en plazos no mayores de 36 hrs. debido a que en periodos mayores de tiempo, se favorece la proliferación bacteriana con el consecuente daño a la integridad aponeurótica y existe mayor posibilidad de desgarro de las fascias y músculo de la pared abdominal.

El cierre secuencial de la pared abdominal puede ser efectuado en la cama del paciente, permitiendo idealmente a corto plazo cerrar de manera definitiva la aponeurosis y el resto de la pared abdominal en un nuevo ingreso a quirófano.

Se decide cerrar en forma definitiva la pared abdominal cuando:

1. La presión abdominal es menor a 20 $\mathrm{mmHg}$.

2. Cuando las fascias se han aproximado lo suficiente para permitir un cierre seguro de la pared abdominal.

3. Cuando existe la seguridad de que no hay fugas intestinales.

4. Cuando la continuidad intestinal ha quedado restablecida y es segura.

5. Cuando no se esperan problemas posteriores de deficiencia en la cicatrización.

6. Cuando se ha logrado la desbridación suficiente de tejido necrótico y se ha controlado la infección intraabdominal. 


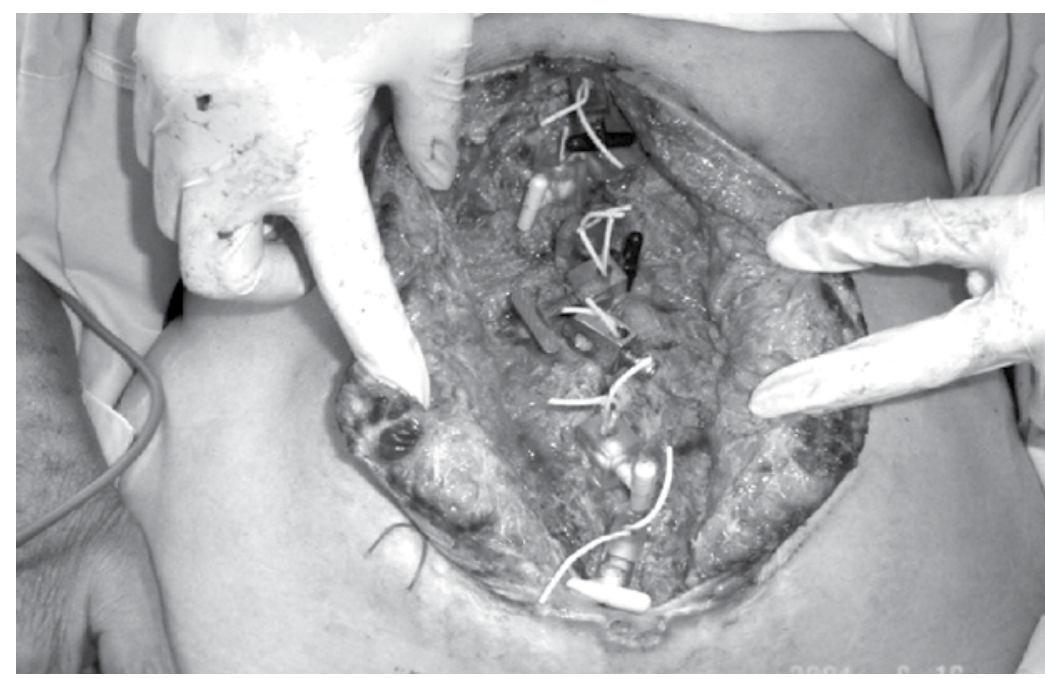

Figura 4. Afrontamiento progresivo de las aponeurosis manteniendo la tracción mediante cubos acrílicos diseñados en el $\mathrm{CHMH}$.

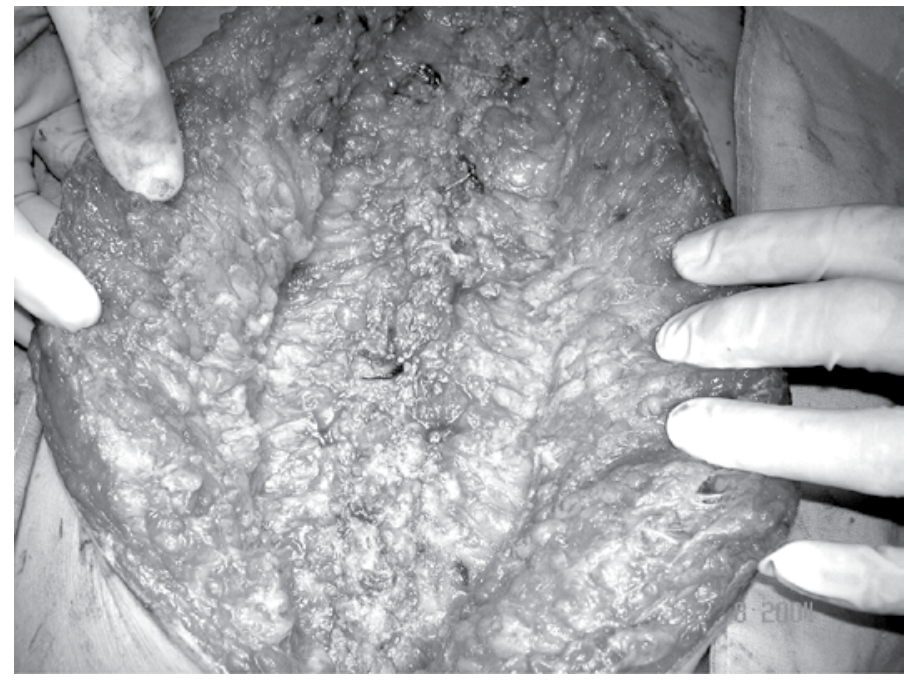

Figura 5. Cierre definitivo de la pared suturando los bordes de la aponeurosis.

\section{Conclusión}

El Cierre Secuencial de la Pared Abdominal es una técnica quirúrgica ideada y empleada en el Centenario Hospital Miguel Hidalgo, de manera protocolizada y estandarizada, en la cual se ofrece una alternativa para el manejo de pacientes con abdomen abierto con resultados iniciales alentadores. 


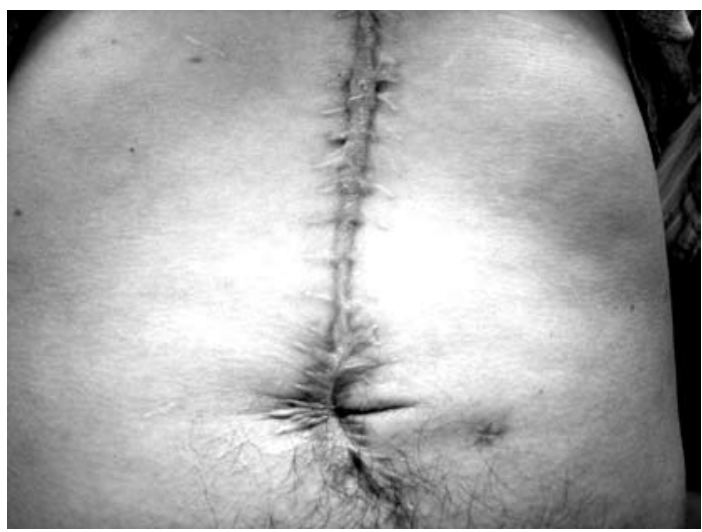

Figura 6. Resultado final en el abdomen de un paciente tratado con cierre secuencia de la pared abdominal.

\section{Bibliografía}

1 Lossanoff JE, Richman BW, Jones JW. Temporary abdominal coverage and reclosure of the open abdomen: Frecuently Asked questions. J Am Coll Surg 2002; 195(1):105-115.

2 De la Fuente LM, Mendoza MV, Robledo OF y cols. Cierre temporal de la pared abdominal con polietileno. Cir Ciruj 2002; 70:157-163.

3 Flores AE, Ávila CG, López RJ, Reynoso TD, De la Torre GJ, Rivera BV. Detección temprana y factores de riesgo asociados al síndrome compartimental abdominal. Cir Ciruj 2005; 73: 179-183.

4 Price J. Surgical intervention in cases of peritonitis. Proc Philadelphia. County Med Soc 1905; 26: 92

5 Torek $F$. The diagnosis and treatment of peritonitis of the upper abdomen. Boston Med J Surg 1910; 162:485.

6 Artz CP, Bornett WO, Grogan JB. Further studies concerning the pathogenesis and treatment of peritonitis. Ann Surg 1962; 155: 756-67.

7 Hudspeth AS. Radical surgical debridement in the treatment of advanced generalized bacterial peritonitis. Arch Surg 1975; 110: 1.233-6.

8 Steinberg D. On leaving the peritoneal cavity open in acuted generalized suppurative peritonitis. Am J Surg 1979;137:216-20. 25.

9 Teichmann W, Wittmann DH, Andreone PA. Scheduled reoperations (etappenlavage) for diffuse peritonitis. Arch Surg. 1986 Feb;121(2):147-152.

10 Borráez O. Abdomen abierto. Utilización del polivinilo. Rev Colomb Cir 2001;16(1):39-43.

11 Ogilvie $\mathrm{WH}$. The late complications of abdominal war wounds. Lancet 1940;2:253-6.

12 Eddy VA, Key SP Mirris JA. Abdominal compartment syndrome: etiology, detection, and management..J Tenn Med Assoc 1994; 55-7.

13 Borráez OA. Abdomen abierto. En: Quintero, Nieto, Lerma, editors. Infección en cirugía. Bogotá, D.C., Ed. Médica Panamericana, 2001.
14 Asher H, Mattox K. Cirugía de control de daños. Clín Quirurg Norte Am. 1997;4:751-948.

15 Wittmann DH. Staged Abdominal reapair: Development and Current Practice of an Advanced Operative technique for diffuse suppurative peritonitis. Eur Surg 2000; 32:171-178.

16 Mayer D, Veith FJ, Lachat M, Pfammatter T, Hechelhammer L, Rancic Z. Abdominal compartment syndrome. Minerva Chir 2010;65(3):329-46.

17 Barker DE, Kaufman HJ et al. Vacuum pack technique of temporary Abdominal closure: a 7-year experience with 112 patients. J Trauma 2000;48:201-207.

18 Schmelzle $M$, Alldinger I, Matthaei $\mathrm{H}$, et al. LongTerm Vacuum-Assisted Closure in Open Abdomen due to Secondary Peritonitis: A Retrospective Evaluation of a Selected Group of Patients. Dig Surg. 2010; $22,27(4): 272-278$

19 Jonathan AM, Barbara AL. Nonoperative progresive "bogota Bag" closure after abdomuinal decompression. Am Surg 2002;68:1229-1231.

20 Konaris LG, Hendrickson RJ, Drugas G. Dynamic retention a technique for closure of the complex abdomen in critically ill patients. Arch Surg 2001;136:1359-1363.

21 Atweh NA, Lye KD, Kavic S, Fidler PE, Possenti $P$, Dudrick SJ. Closure of large abdominal wounds with an adjustable suture-tension device. J Am Coll Surg 2002;195(2):114-117.

22 Fansler R, Taheri $\mathrm{P}$ et al. Polypropylene mesh closure of the complicated abdominal wound. Am J Surg 1995;170:15-18.

23 Miller PR, Thompson JT, Faler BJ, Meredith JW. Late fascial Closure in leu of Ventral hernia: The Next Step in open abdomen management. J Trauma 2002;53:843-849.

24 Flores AE, Avila GG, de la Torre GJC, Rivera BV, López RJL, Reynoso TD. Diagnóstico temprano y factores de riesgo asociados al Síndrome Compartimental Abdominal. Cir Cir 2005;73:179-183. 